\title{
Peripheral refraction in myopic patients after orthokeratology ${ }^{\dagger}$
}

\begin{abstract}
Objective: The purpose of this study was to characterize the central and peripheral refraction across the horizontal meridian of the visual field before and after myopic corneal refractive therapy (CRT) with contact lenses.
\end{abstract}

Methods: Twenty-eight right eyes (mean age $\pm S D=24,6 \pm 6,3$ years) were fitted with Paragon CRT contact lenses to treat myopia between $-0,88$ and $-5,25 \mathrm{D}$ of spherical equivalent. Along with a complete set of examination procedures to assess suitability for treatment, the central and peripheral refraction was measured along the horizontal meridian up to $35^{\circ}$ of eccentricity in the nasal and temporal retinal area in $5^{\circ}$ steps.

Results: Baseline central average spherical equivalent (M) measured by subjective refraction changed from $-1,95 \pm 1,27 D$ to $-0,38 \pm 0,67 D$. Changes in $M$ component ranged between $1,42 \pm 0,89 \mathrm{D}$ at center to $0,43 \pm 0,88 \mathrm{D}$ at $20^{\circ}$ in the temporal retina $(p<0,002)$. At $25^{\circ}$ to both sides of the central refraction measurement, peripheral refraction after treatment was not statistically different from baseline values $(p>0,351)$. Beyond the $25^{\circ}$ limit, $M$ component changed in the myopic direction up to $-1,11 \pm 0,88 \mathrm{D}$ at $35^{\circ}$ in temporal retina $(p<0,001)$. Treatment induced was symmetric between nasal and temporal visual field along the horizontal meridian $(p>0,05$ for all eccentricities). Furthermore, the degree of myopic increase in spherical equivalent for $30^{\circ}$ $(r 2=0,573, p<0,001)$ and $35^{\circ}(r 2=0,645, p<0,001)$ eccentric refraction was highly correlated with axial spherical equivalent at baseline.

Conclusions: CRT inverts the pattern of peripheral refraction in spherical equivalent refraction creating a treatment area of myopic reduction within the central $25^{\circ}$ of visual field, and a myopic shift beyond the $25^{\circ}$. In peripheral refraction for $30^{\circ}$ and $35^{\circ}$, the amount of myopia induced in terms of spherical equivalent has an almost 1:1 relationship with the amount of baseline spherical equivalent refraction to be corrected.

+Queirós A., González-Méijome J.M., Jorge J., Villa-Collar C., Gutiérrez A.R. Peripheral Refraction in Myopic Patients after Orthokeratology. Optometry and Vision Science. 2010;87(5): 323-329. 


\section{Introduction}

Myopia affects approximately $25 \%$ of the World population and has become a public health concern due to the socioeconomic impact and to the risk of vision loss related to other comorbidities. ${ }^{1}$ For these reasons there is great interest in solutions to prevent myopia onset and progression. However, such approaches will need to be based on the mechanisms involved to try to interfere with them in a desirable way.

Different etiological factors have been proposed for myopia onset and progression, such as an increase in intraocular pressure, ${ }^{2}$ anomalous accommodative activity ${ }^{3}$ and defocus of the retinal image. ${ }^{4}$ Depending on the implicated mechanism, different clinical approaches have been developed including the use of drugs to lower $\mathrm{IOP}^{5}$, relaxation of accommodation with atropine ${ }^{6}$ or pirenzepine, ${ }^{7}$ monofocal, bifocal or multifocal spectacle lenses, ${ }^{8.10}$ or conventional geometry, ${ }^{11}$ or reverse geometry rigid gas-permeable contact lenses. ${ }^{12}$

Given the lack of effect on axial elongation of conventional geometry lenses, ${ }^{11}$ the most promising approach involving contact lenses for slowing myopia progression consists of myopia correction with reverse geometry contact lenses for corneal reshaping. Several studies have shown lower annual increase of vitreous chamber depth and myopia progression in children wearing these lenses when compared with groups wearing spectacle lenses, ${ }^{12}$ soft contact lenses. ${ }^{13}$

The hypothesized reason for corneal reshaping interfering with the ocular growth pattern is that it induces a myopic change in relative peripheral refractive error (RPRE) while the central refraction is fully corrected. According to this theory, the myopic RPRE following CRT prevents the hyperopic RPRE usually present in the myopic eye from acting as a stimulus for ocular growth, as has been suggested in animal models. ${ }^{1416}$ Bakaraju et al. have suggested that the risk for myopia progression could be higher in axial myopias compared to refractive ones according to their peripheral refractive pattern. ${ }^{17}$

Despite these findings, the characterization of peripheral refraction after corneal reshaping has only been addressed by a limited number of studies. ${ }^{18}$ In the work of Charman et al., the authors showed for the first time the actual changes in off-axis refraction induced by ortho-k treatment. Furthermore, new studies are evaluating the impact of the treatment in off-axis aberrations confirming that orthokeratology increases the aberrations when measured with eccentric fixation..$^{19}$ Another study conducted in our group showed that corneal first surface aberration increases exponentially after orthokeratology with the increase in pupil size (Queiros et 
al, submitted for publication). In this study we report clinical data for the peripheral refraction after corneal reshaping in eyes whose original refractions covered most of the range currently approved by the FDA for this treatment and using a different lens design than that used in the study conducted by Charman and coworkers. ${ }^{18}$ In addition, the present study shows a statistical approach to evaluate the symmetry of refractive profiles to both sides of the peripheral visual field across the horizontal meridian.

\section{Methods}

\section{Subjects and inclusion criteria}

Measurements were made on twenty-eight right eyes of 28 university students with a mean age of $24,6 \pm 6,3$ years (ranging from 20 to 41), of which 11 were female $(39,3 \%)$ and 17 were male $(60,7 \%)$. Total preoperative spherical equivalent obtained with subjective refraction was $1,95 \pm 1,27 \mathrm{D}$ (from $-0,88$ to $-5,25 \mathrm{D}$ ). After explaining the nature of the study, each patient signed a consent form before being enrolled. The research followed the tenets of the Declaration of Helsinki and was reviewed and approved by the Scientific Committee of the School of Sciences of Minho University (Portugal). The inclusion criteria required that the subjects did not suffer from any current eye disease or injury and were not taking any ocular or systemic medication.

Subjective non-cycloplegic refraction was performed monocularly. The criteria of maximum plus for best visual acuity was used to arrive to the end point of refraction. The intraocular pressure was checked with a non-contact tonometer before and after treatment (Nidek Model NT-4000, noncontact tonometer)..$^{20}$

\section{Peripheral Refraction}

The measurement of central and peripheral refraction was obtained with the open-field Grand Seiko Auto-Refractometer/Keratometer WAM-5500 (Grand Seiko Co., Ltd., Hiroshima, Japan). The instrument has been previously used and validated to measure refraction in the centra $^{21}$ and peripheral retina. ${ }^{22}$ The system was attached to software created to automatically record data from the autorefractometer thus avoiding errors in data collection and allowing data to 
be automatically processed in an Excel spreadsheet for later statistical analysis process using appropriate software.

The illumination of the room was adjusted to obtain a pupil size greater than $4 \mathrm{~mm}$ required to allow peripheral measurements, which was achieved in all cases. The fixation target was placed at a distance of 2,5 meters from the patient's corneal vertex and consisted of 15 LEDs in the horizontal direction: one central, seven to the right and seven to the left side. The LEDs were separated from each other by an angular distance of $5^{\circ}$ at the patient's position. The subject was seated with the head stabilized in a chin-rest so that the eye was aligned with the central LED. For the right eye, the fixation of an object positioned on the right side of the central point (nasal visual field in the eye primary position) matches the temporal retina measures. The left eye was occluded while patients kept their head stationary and rotated their right eyes to view a series of fixation targets. Five readings were taken and averaged only on the right eye of each individual in all positions. The axis of the autorefractor was aligned with the center of the entrance pupil during all measurements.

Descriptive statistics (mean \pm S.D.) were obtained for the refraction vector components,

$$
\begin{gathered}
M=S p h+C y / / 2, \\
J 0=-C y / \cdot \cos (2 \theta) / 2 \text { and } \\
J 45=-C y / \cdot \sin (2 \theta) / 2
\end{gathered}
$$

according to Fourier analysis, as recommended by Thibos,,$^{23}$ where Sph, Cyl and $\Theta$ are the manifest sphere, cylinder and axis, respectively.

\section{Corneal refractive therapy lens characteristics}

Paragon CRT (paflufocon D, Dk=100 barrer) sigmoid reverse geometry rigid gas permeable lenses were used (Paragon Vision Sciences, Mesa, AZ, USA). Trial lenses were derived from sliding table nomograms provided by the manufacturer and which have shown high levels of predictability in terms of first trial success. ${ }^{24}$ Fitting was evaluated according to the recommendations of the manufacturer regarding fluorescein pattern, topographical evaluation, refractive and visual outcomes. Parameters of the CRT ${ }^{\top m}$ lenses were (mean \pm S.D. [minimum, maximum]), base curve radius: ( $B C R=8,38 \pm 0,29 \mathrm{~mm}[7,90 ; 9,00 \mathrm{~mm}])$, return zone depth: 
(RZD $=530,88 \pm 18,52 \mu \mathrm{m}[500 ; 575 \mu \mathrm{m}])$ and landing zone angle: (LZA=32,85 $\pm 0,66$ degrees $[31,00 ; 34,00$ degrees]). These refer to the final parameters of lenses worn by patients, not necessary the first trial lenses. The number of changes in fitting parameters to achieve the final optimal fitting is well in agreement with previous results obtained in a larger sample size by us using the same lens fitting set. ${ }^{24}$

A minimum treatment period of one month was required to guarantee that the treatment was completely stable. ${ }^{25}$ The time between pre and post treatment measures was $37,0 \pm 3,0$ days. During that period, lenses were worn overnight for $7,82 \pm 1,02$ hours. After the first night of treatment where the patients attended the clinic wearing their lenses, they were asked to insert the lenses ten minutes before sleep along with a drop of artificial tear. The patients removed the lenses within ten minutes after waking-up in the following morning after applying again a drop of artificial tear solution. The measurements were performed between 9:00 and 11:00, A.M. and at least 2 hours after lens removal to minimize the influence of treatment regression ${ }^{26}$ and diurnal variations in corneal thickness that might potentially influence anterior corneal topography. ${ }^{27,28}$

\section{Statistical analysis}

The SPSS software package v.17 (SPSS Inc., Chicago, IL, USA) was used for statistical analysis. Kolmogorov-Smirnov Test was applied in order to evaluate the normality of the data distribution. When normality could not be assumed, the Wilcoxon Signed Ranks Test was used for paired comparison post and pretreatment and Paired Samples t-Test was used when normality could be assumed for pair comparisons between treatments. For statistical purposes, a $p$ value lower than 0,05 was considered statistically significant.

\section{Results}

Table 1 presents descriptive statistics for refractive components $\mathrm{M}$, J0 and J45 in the center of the visual field at baseline and after treatment as well as comparisons among them. Statistically significant differences were found for the spherical equivalent (diff post-pre= $+1,57 \pm 0,77 \mathrm{D}, p<0,001$, Wilcoxon Signed Ranks Test), but not for the astigmatic components J0 ( $p=0,778$, Wilcoxon Signed Ranks Test) and J45 ( $p=0,422$, Paired Samples t-Test). 
Table 1 - Demographic characteristics (mean \pm S.D.) of the population in the baseline and post treatment, the mean difference and the correlation between them (refraction error obtained with subjective refraction for central refraction)

\begin{tabular}{lcccc}
\hline $\mathbf{n}=\mathbf{2 8}$ & Pré & Pós & Pós - Pré & p \\
\hline Esferra (D) & $-1,73 \pm 1,22$ & $-0,14 \pm 0,61$ & $+1,60 \pm 0,77$ & $<0,001^{*}$ \\
Cilindro (D) & $-0,43 \pm 0,33$ & $-0,49 \pm 0,31$ & $-0,06 \pm 0,31$ & $0,274^{\text {s }}$ \\
M (D) & $-1,95 \pm 1,27$ & $-0,38 \pm 0,67$ & $+1,57 \pm 0,77$ & $<0,001^{s}$ \\
J0 (D) & $+0,05 \pm 0,21$ & $+0,06 \pm 0,22$ & $+0,01 \pm 0,23$ & $0,778^{s}$ \\
J45 (D) & $+0,01 \pm 0,16$ & $+0,04 \pm 0,18$ & $+0,03 \pm 0,20$ & $0,422^{*}$ \\
\hline
\end{tabular}

*Paired Samples t-Test, sWilcoxon Signed Ranks Test.

Vector analysis of refractive components is illustrated in figure 1 for baseline data and after treatment. Spherical equivalent refraction shows a significant myopic reduction within the central $40^{\circ}$, being maximum at the center. At $25^{\circ}$ however, the treatment showed no effect on the $\mathrm{M}$ component, while a myopic increase is noticed beyond the central $50^{\circ}$ of the visual field $\left(25^{\circ}\right.$ along each semi-meridian to the nasal and temporal sides of the central measurement). For astigmatism components, again no significant differences exist for the J0 component within the central $20^{\circ}$ (10 $0^{\circ}$ to each side), but a significant increase in myopic astigmatism is observed beyond this point. This component increases about $-0,50 \mathrm{D}$ or beyond this value for eccentricities beyond $20^{\circ}$ on each side of the visual field. Conversely, no consistent significant changes were noticed across the field for the J45 component.

Comparing points located symmetrically to both sides of the central refraction there was not statistically significant differences in M component $(p>0,090)$. Same result was found when the differences between baseline and post-treatment refraction are compared between symmetrical angles of eccentric refraction $(p>0,050)$. This means that CRT treatment renders symmetrical patterns of refraction to both sides of the central visual field along the horizontal meridian. 

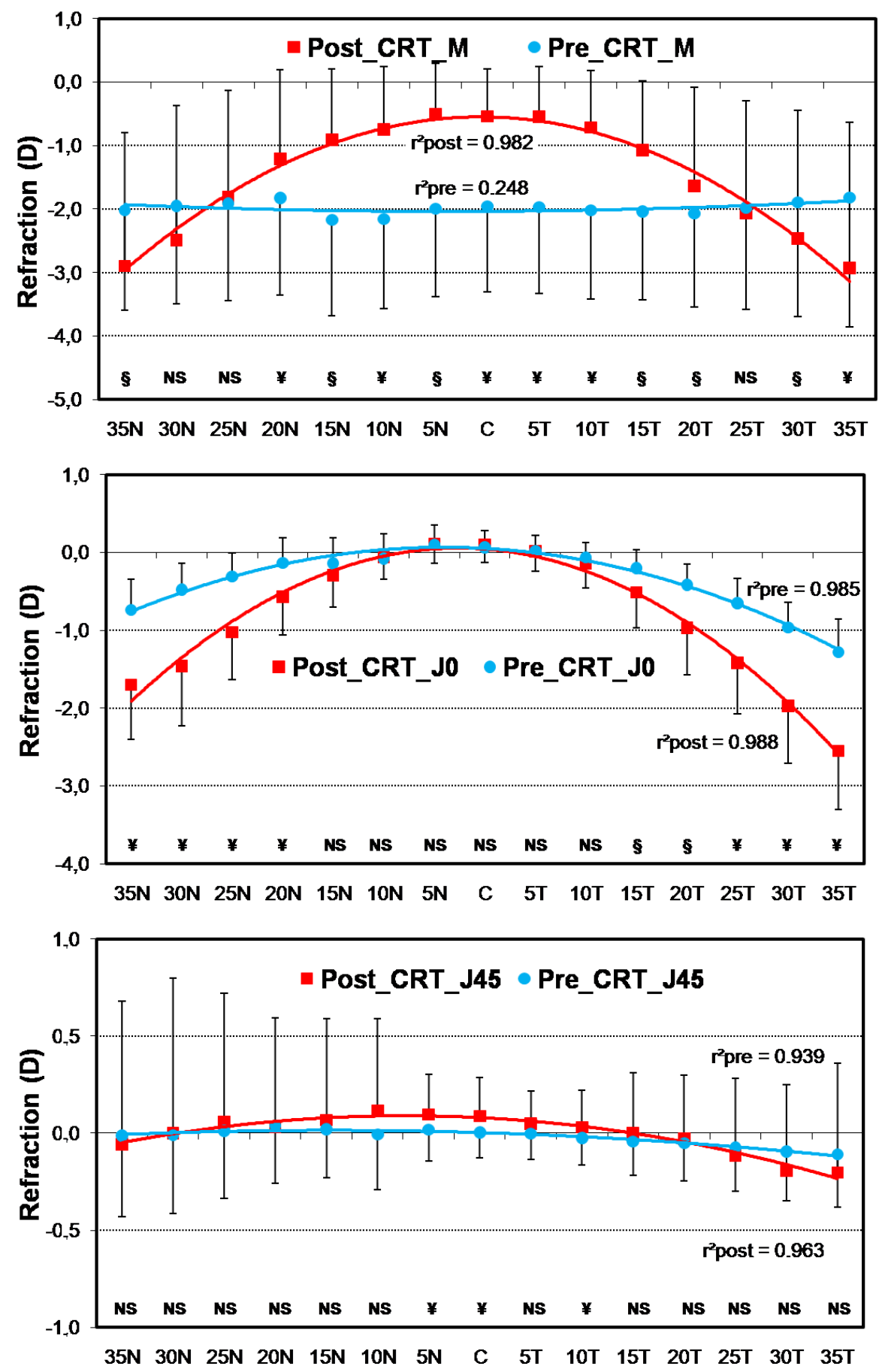

Figure 1 - Spherical equivalent $\mathrm{M}$ and cylindrical components $\mathrm{J} 0$ and $\mathrm{J} 45$ refraction as a function of field angle in orthokeratology patients in the temporal (T) and nasal (N) retinal area, at baseline (grey circles), and post-treatment (black squares). Bars represent standard deviation. Statistical significant differences: $¥$ Paired Samples t-Test, § Wilcoxon Signed Ranks Test, NS - nonsignificant values. Lines represent the 2 th order polynomial fit for $M\left(y_{\text {pos }}=-0,0510 x^{2}+0,8037 x-\right.$ $3,7149, \quad r^{2}=0,982$ and $\left.y_{\text {pre }}=+0,0028 x^{2}-0,0399 x-1,8941, \quad r^{2}=0,248\right) ; \quad$ for J0 $\left(y_{\text {pos }}=-\right.$ $0,0468 x^{2}+0,7007 x-2,5662, r^{2}=0,988$ and $\left.y_{\text {pre }}=-0,0215 x^{2}+0,3098 x-1,0478, r^{2}=0,985\right)$ and for $J 45$ $\left(y_{\text {pos }}=-0,0045 x^{2}+0,0589 x-0,1032, r^{2}=0,963\right.$ and $\left.y_{\text {pre }}=-0,0013 x^{2}+0,0135 x-0,0198, r^{2}=0,939\right)$. 
Figure 2 represents a summary of the relative changes of refraction compared to baseline for different components of refraction $\mathrm{M}, \mathrm{J} 0$ and J45. These data represent relative values of refraction where all the curve was shifted to set the central refractive value at "zero"; this enhances the visibility of the relative changes in peripheral refraction compared to central measurement. On average, about $-2,5 \mathrm{D}$ of relative peripheral spherical equivalent is induced by CRT. Of those, approximately $-1,5 \mathrm{D}$ of this change is due to changes in the sphere and the other $-1,0 \mathrm{D}$ is due to the $-2,0 \mathrm{D}$ change in the cylinder.

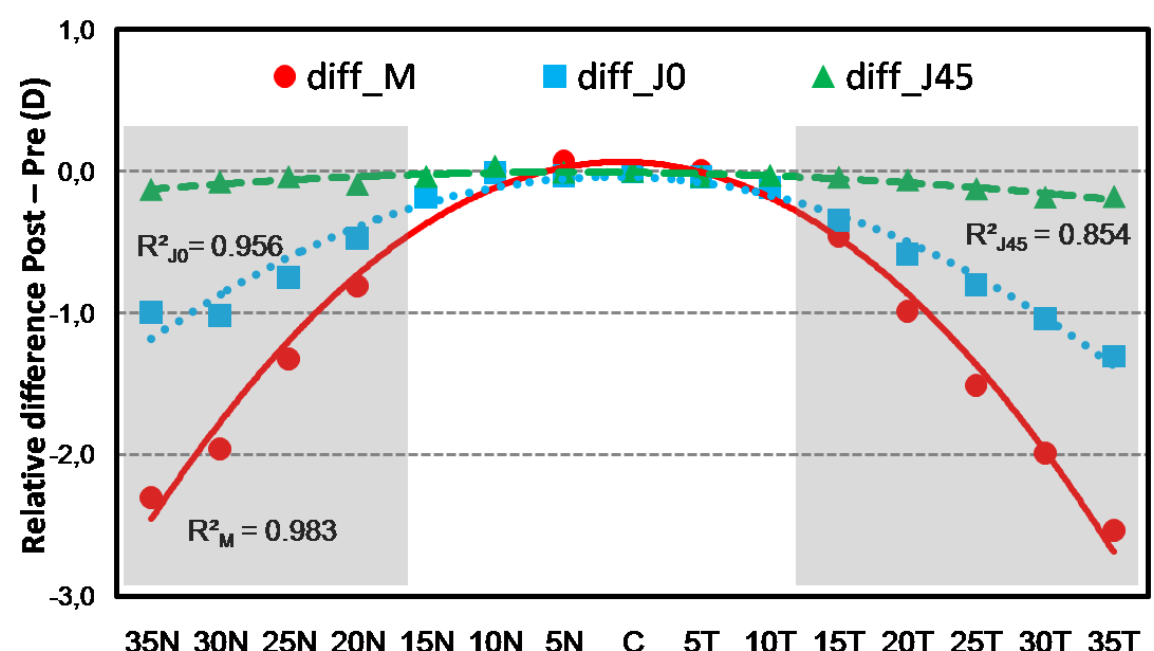

Figure 2 - Relative differences (post minus pre treatment) in components of refraction as a function of field angle in orthokeratology patients in the temporal (T) and nasal (N) retinal area. The shaded area represents the points where the changes in off-axis spherical equivalent (M) are significantly more myopic $(p<0,050)$ than those induced in the central visual axis. Lines represent the 2 th order polynomial fit for $M\left(y_{d i f}=-0,0538 x^{2}+0,8436 x-3,2424, r^{2}=0,983\right)$; for J0 $\left(y_{d i f}=-0,0253 x^{2}+0,3909 x-\right.$ $\left.1,5507, r^{2}=0,956\right)$ and for J45 ( $\left.y_{\text {dif }}=-0,0032 x^{2}+0,0454 x-0,1684, r^{2}=0,854\right)$.

When each eccentric point was compared to central measurement, results showed that differences in relative spherical equivalent $M$ among different eccentric points became only statistically significant (i.e., where does the significant relative peripheral myopia start), for nasal retina beyond 15 degrees (i.e. $p<0,05$ only for $20^{\circ}$ and beyond according to Wilcoxon Signed Ranks Test), and for temporal retina beyond 10 degrees (i.e. $p<0,05$ only for $15^{\circ}$ and beyond according to Wilcoxon Signed Ranks Test). Regarding changes in J45, none of the points analyzed except two locations $\left(20^{\circ}\right.$ nasal and $30^{\circ}$ temporal) were statistically different from axial refraction. 
Regarding changes in J0 component, all points except central ones ( $15^{\circ}$ nasal to $5^{\circ}$ temporal) showed statistically significant differences when compared with changes in axial refraction.

Figure 3 shows the degree of peripheral myopia induced by CRT (average values from nasal and temporal locations) as a function of individual baseline $\mathrm{M}$ component. This analysis showed that peripheral values obtained at $30^{\circ}\left(r^{2}=0,573, p<0,001\right)$ and $35^{\circ}\left(r^{2}=0,645, p<0,001\right)$ reflect the higher correlation with baseline spherical equivalent refraction. Interestingly, when data from the two outlier present in the graph was removed, the correlations increased to $\left(r^{2}=0,686\right.$, $p<0,001)$ for $30^{\circ}$ and $\left(r^{2}=0,771, p<0,001\right)$ for $35^{\circ}$.

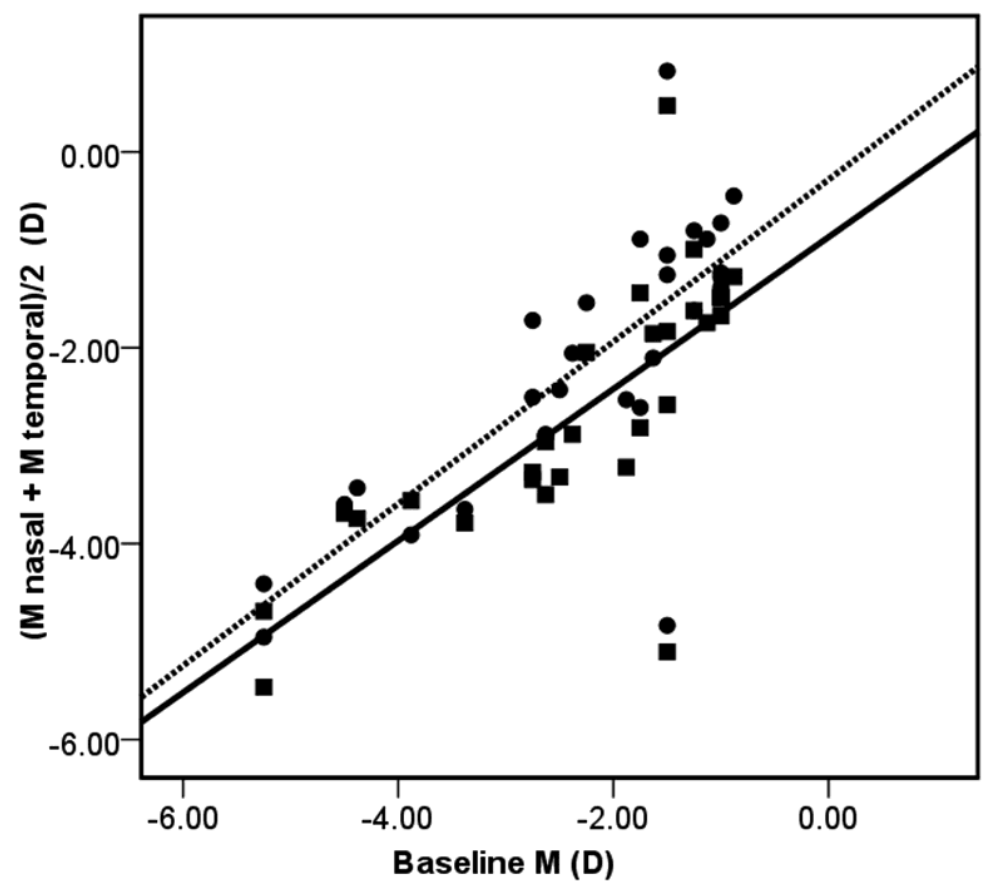

Figure 3 - Change in spherical equivalent after CRT for a given peripheral eccentric location [(M nasal $+M$ temporal)/2] as a function of the axial spherical equivalent at baseline. For clarity, only the higher correlations are shown, corresponding to the $30^{\circ}\left(r^{2}=0,573, y=0,8279 x-0,282\right.$, circles, point line, ) and $35^{\circ}\left(r^{2}=0,645, y=0,7746 x-0,8719\right.$, squares, full line) eccentric locations.

For those locations, there was a linear regression line that describes a nearly $1: 1$ relationship between average change in spherical equivalent for a given peripheral eccentric refraction (homologous nasal and temporal locations) and the axial spherical equivalent at baseline. The remaining correlations, despite still being statistically significant, were progressively lower from $r^{2}=0,489$ for $25^{\circ}$ eccentricity to $r^{2}=0,026$ for $5^{\circ}$ eccentricity (table 2 ). 
Table 2 - Correlations between change in spherical equivalent after CRT for a given peripheral eccentric location [(M nasal $+M$ temporal)/2] and the axial spherical equivalent at baseline $(n=28$ olhos)

\begin{tabular}{ccc}
\hline Eccentricity & Correlation $\left(\mathbf{r}^{\mathbf{2}}\right)$ & $\mathbf{p}$ (significance) \\
\hline 35 degrees & 0,645 & $<0,001$ \\
30 degrees & 0,573 & $<0,001$ \\
25 degrees & 0,482 & $<0,001$ \\
20 degrees & 0,349 & $<0,001$ \\
15 degrees & 0,116 & 0,049 \\
10 degrees & 0,092 & 0,081 \\
5 degrees & 0,026 & 0,364 \\
\hline
\end{tabular}

\section{Discussion}

Corneal reshaping with contact lenses has experienced a rebirth in recent years motivated by the development of high permeability to oxygen materials and the advances in the contact lens designs. ${ }^{12,29313}$ At the same time, the interest in CRT was renewed as one of the most promising techniques to reduce the progression of myopia, particularly by suggestions that it can slow myopia progression in children.12,32 This reduction would be caused by the alteration of power distribution between the central and paracentral region of the cornea that provides the desired myopic stimulus in the parafoveal region while the central region remains focused.

The present study shows the refractive changes across the horizontal $70^{\circ}$ of the visual field in a sample of myopic patients representative of the current application ranges for corneal refractive therapy using contact lenses (CRT). Results have shown that true refractive correction is achieved for spherical refraction within the central $20^{\circ}$ of the visual field (10 degrees to the nasal and $10^{\circ}$ to the temporal field). The cylindrical refraction does not change in a clinically significant way within the same area compared to baseline. Beyond the central $20^{\circ}$, myopia is not totally reduced and at $35^{\circ}$ to each side of the visual field sphere did not change compared to baseline. Sphere equivalent refraction shows no change compared to baseline at $25^{\circ}$ to each side of the 
temporal visual field and beyond this point this component remains myopic, particularly due to the increase in cylinder (with minus sign).

A previous study has shown that this can be accomplished with orthokeratology in a limited sample of patients ${ }^{18}$ and a single design or lens. This is now confirmed in a much larger patient group whose original refractions cover almost the entire range of application of ortho-K( up to $6,00 \mathrm{D})$, and using a different design of FDA-approved lens.

The symmetry achieved in the refractive pattern relative to the center of the visual field is noteworthy. This reflects the good centration and predictability achieved with modern orthokeratology lenses ${ }^{24}$ as we have already showed in the topographic analysis of changes in curvature profiles after same treatments..$^{33}$ This centration also suggests good stability of astigmatic components in the center of the visual field, while the peripheral visual field beyond the central 20 degrees suffers a significant increase in astigmatism after CRT treatment.

Regarding the changes in peripheral refraction achieved by CRT treatment, and considering that a myopic RPRE would be desirable for the purposes of control of myopia progression, the present results show that the changes are quite complex. Apparent relative peripheral myopization does not reflect an increase in spherical component which would lead to a true myopization. Instead, what happens is that myopia is fully corrected within the central $20^{\circ}$ of the visual field and beyond this point myopia correction (in terms of spherical refraction) starts to decrease up to $35^{\circ}$ where no significant change is detected. Part of the increase in myopia in terms of spherical equivalent is induced by the increase in astigmatic refraction (about -1D that in terms of $M$ value represents about $-2 \mathrm{D}$ of cylinder). This increase in astigmatism could be related with astigmatic refraction experienced by oblique rays passing through the margins of the treatment zone, also known "steepening ring" coincidental with the reverse curve of the contact lens. In our opinion, this reflects the astigmatic optical effect experienced by the light passing through the steepening ring surrounding the optical zone that acts as a convex (positive) cylindrical lens for the given light beam.

Another interesting outcome is that as regression lines showed in figure 3 , for $30^{\circ}$ and $35^{\circ}$, the amount of myopia induced in terms of spherical equivalent has an almost 1:1 relationship with the amount of baseline spherical equivalent refraction to be corrected.

In summary, considering manifest refraction in clinical notation, changes in sphere component cover the whole visual field, except for the most peripheral locations at $35^{\circ}$ in each side of the visual field; conversely, cylinder remained unchanged at the center of the visual field, but increased 
significantly beyond the central $20^{\circ}\left(10^{\circ}\right.$ to each side of the visual field). Considering the spherical equivalent, significant myopic reduction is achieved within the central $40^{\circ}\left(20^{\circ}\right.$ to each side), no changes occurred at $25^{\circ}$ and a significant increase of myopia was observed beyond the $25^{\circ}$ location.

\section{ACKNOWLEDGMENTS:}

SUPPORTED BY A GRANT (\#SFRH/BD/61768/2009) FROM THE SCIENCE AND TECHNOLOGY FOUNDATION OF PORTUGUESE. Ministry of Science and Superior Education (European Social Funding). We have no proprietary interest in any of the instruments or materials mentioned in this article. The authors want to thank Paragon Vision Science (Mesa. AZ) e Interlenco SA (Madrid. Spain) for donating the lenses and Bausch \& Lomb (Rochester. NY) for donating the care solutions used in the study

\section{Referências}

1. Kempen JH, Mitchell P, Lee KE, Tielsch JM, Broman AT, Taylor HR, Ikram MK, Congdon NG, O'Colmain BJ. The prevalence of refractive errors among adults in the United States, Western Europe, and Australia. Arch Ophthalmol 2004; 122: 495-505.

2. Goss DA ,Caffey TW. Clinical findings before the onset of myopia in youth: 5. Intraocular pressure. Optom Vis Sci 1999; 76: 286-291.

3. Chen JC, Schmid KL, Brown B. The autonomic control of accommodation and implications for human myopia development: a review. Ophthalmic Physiol Opt 2003; 23: 401-422.

4. Atchison DA, Pritchard N, Schmid KL. Peripheral refraction along the horizontal and vertical visual fields in myopia. Vision Res 2006; 46: 1458.

5. Jensen H. Myopia progression in young school children. A prospective study of myopia progression and the effect of a trial with bifocal lenses and beta blocker eye drops. Acta Ophthalmol Suppl 1991; 1-79.

6. Lee JJ, Fang PC, Yang IH, Chen CH, Lin PW, Lin SA, Kuo HK, Wu PC. Prevention of myopia progression with 0.05\% atropine solution. J Ocul Pharmacol Ther 2006; 22: 41-46.

7. Tan DT, Lam DS, Chua WH, Shu-Ping DF, Crockett RS. One-year multicenter, double-masked, placebo-controlled, parallel safety and efficacy study of $2 \%$ pirenzepine ophthalmic gel in children with myopia. Ophthalmology 2005; 112: 84-91.

8. Gwiazda J, Hyman L, Hussein M, Everett D, Norton TT, Kurtz D, Leske MC, Manny R, MarshTootle W, Scheiman M. A randomized clinical trial of progressive addition lenses versus 
single vision lenses on the progression of myopia in children. Invest Ophthalmol Vis Sci 2003; 44: 1492-1500.

9. Fulk GW, Cyert LA, Parker DE. A randomized trial of the effect of single-vision vs. bifocal lenses on myopia progression in children with esophoria. Optom Vis Sci 2000; 77: 395-401.

10. Edwards MH, Li RW, Lam CS, Lew JK, Yu BS. The Hong Kong progressive lens myopia control study: study design and main findings. Invest Ophthalmol Vis Sci 2002; 43: 28522858.

11. Walline JJ, Jones LA, Mutti DO, Zadnik K. A randomized trial of the effects of rigid contact lenses on myopia progression. Arch Ophthalmol 2004; 122: 1760-1766.

12. Cho $P$, Cheung SW, Edwards $M$. The longitudinal orthokeratology research in children (LORIC) in Hong Kong: a pilot study on refractive changes and myopic control. Curr Eye Res 2005; 30: 71-80.

13. Walline JJ, Jones LA, Sinnott LT. Corneal Reshaping and Myopia Progression. Br J Ophthalmol 2009.

14. Smith EL, III, Kee CS, Ramamirtham R, Qiao-Grider Y, Hung LF. Peripheral vision can influence eye growth and refractive development in infant monkeys. Invest Ophthalmol Vis Sci 2005; 46: 3965-3972.

15. Smith EL, III, Ramamirtham R, Qiao-Grider Y, Hung LF, Huang J, Kee CS, Coats D, Paysse E. Effects of foveal ablation on emmetropization and form-deprivation myopia. Invest Ophthalmol Vis Sci 2007; 48: 3914-3922.

16. Diether $S$,Schaeffel $F$. Local changes in eye growth induced by imposed local refractive error despite active accommodation. Vision Res 1997; 37: 659-668.

17. Bakaraju RC, Ehrmann K, Papas EB, Ho A. Do peripheral refraction and aberration profiles vary with the type of myopia? - An illustration using a ray-tracing approach. J Optom 2009; 2: 29-38.

18. Charman WN, Mountford J, Atchison DA, Markwell EL. Peripheral refraction in orthokeratology patients. Optom Vis Sci 2006; 83: 641-648.

19. Mathur A ,Atchison DA. Effect of orthokeratology on peripheral aberrations of the eye. Optom Vis Sci 2009; 86: E476-E484.

20. Queiros A, Gonzalez-Meijome JM, Fernandes P, Jorge J, Almeida JB, Parafita MA. Noncontact tonometry synchronized with cardiac rhythm and its relationship with blood pressure. Ophthalmic Physiol Opt 2006; 26: 384-391.

21. Queiros A, Gonzalez-Meijome JM, Jorge J. Influence of fogging lenses and cycloplegia on open-field automatic refraction. Ophthalmic Physiol Opt 2008; 28: 387-392.

22. Queiros A, Jorge J, Gonzalez-Meijome JM. Influence of fogging lenses and cycloplegia on peripheral refraction. J Optom 2009; 2: 83-89. 
23. Thibos LN, Wheeler W, Horner D. Power vectors: an application of Fourier analysis to the description and statistical analysis of refractive error. Optom Vis Sci 1997; 74: 367-375.

24. Gonzalez-Meijome JM ,Villa-Collar C. Nomogram, corneal topography, and final prescription relations for Corneal Refractive Therapy. Optom Vis Sci 2007; 84: 59-64.

25. Lu F, Simpson T, Sorbara L, Fonn D. The relationship between the treatment zone diameter and visual, optical and subjective performance in Corneal Refractive Therapy lens wearers. Ophthalmic Physiol Opt 2007; 27: 568-578.

26. Villa-Collar C, Gonzalez-Meijome JM, Queiros A, Jorge J. Short-term corneal response to corneal refractive therapy for different refractive targets. Cornea 2009; 28: 311-316.

27. Handa T, Mukuno K, Niida T, Uozato H, Tanaka S, Shimizu K. Diurnal variation of human corneal curvature in young adults. J Refract Surg 2002; 18: 58-62.

28. Du TR, Vega JA, Fonn D, Simpson T. Diurnal variation of corneal sensitivity and thickness. Cornea 2003; 22: 205-209.

29. Lui WO, Edwards MH, Cho P. Contact lenses in myopia reduction - from orthofocus to accelerated orthokeratology. Cont Lens Anterior Eye 2000; 23: 68-76.

30. Alharbi A, Swarbrick HA. The effects of overnight orthokeratology lens wear on corneal thickness. Invest Ophthalmol Vis Sci 2003; 44: 2518-2523.

31. Sorbara L, Fonn D, Simpson T, Lu F, Kort R. Reduction of myopia from corneal refractive therapy. Optom Vis Sci 2005; 82: 512-518.

32. Cheung SW, Cho P, Fan D. Asymmetrical increase in axial length in the two eyes of a monocular orthokeratology patient. Optom Vis Sci 2004; 81: 653-656.

33. Queiros A, Gonzalez-Meijome JM, Villa-Collar C, Gutierrez AR, Jorge J. Local steepening in peripheral corneal curvature after corneal refractive therapy and LASIK. Optom Vis Sci 2010; 87: 432-439. 